\title{
Diagnóstico Pré-Natal de Fenda Labial e Palatina: Experiência de 40 Casos
}

\author{
Prenatal Diagnosis of Lip and Palate Cleft: Experience of 40 Cases
}

Victor Bunduki, Rodrigo Ruano, Andréia David Sapienza, Beatriz Yae Hanaoka, Marcelo Zugaib

\begin{abstract}
RESUM0
Objetivos: avaliar casos de fenda facial fetal quanto ao tipo de lesão, associação com outras malformações e aneuploidias.

Métodos: as fendas faciais foram estudadas quanto a idade materna e antecedentes, idade gestacional no diagnóstico, lado da lesão, tipo de lesão, presença de malformações associadas e/ou aneuploidias, mortalidade e seguimento pós-natal.

Resultados: em 40 fetos com fenda, a lesão era labial pura em 18 casos (45\%), labiopalatina em 19 (47,5\%) e palatina em 3 (7,5\%). Em 10 casos a fenda era isolada (25\%), todas unilaterais. No grupo de malformações associadas, a aneuploidia esteve presente em 10/30 (33,3\%). A lesão labiopalatina predominou neste grupo (18/30 - 60\%), seguida por fenda bilateral (8/ 30 - 26,7\%) e mediana (10/30 - 33,3\%).

Conclusões: as fendas faciais constituíram excelente marcador para malformações associadas $e$ aneuploidias fetais. Casos de fendas faciais devem ser encaminhados para centros especializados para que se realizem adequados exames ultra-sonográfico e genético tecendo em seguida a conduta. O caráter isolado da fenda facial esteve associado a excelente prognóstico.
\end{abstract}

PALAVRAS-CHAVE: Fenda labial. Fenda palatina. Diagnóstico pré-natal. Malformações fetais.

\section{Introdução}

A fenda labial é a anomalia do maciço ósseo facial mais freqüente ao nascimento. Ela pode ser completa ou incompleta, unilateral ou bilateral, simétrica ou assimétrica e estar associada ou não à fenda palatina. Apesar de muitos casos de fendas faciais não cursarem com outros defeitos ou problemas genéticos associados, sabe-se que o prognóstico fetal se altera profundamente quando estão presentes outras malformações associadas ou aberrações cromossômicas ou, ainda, doenças gênicas ${ }^{1,2}$.

Existem controvérsias quanto à prevalência de associações malformativas ou anomalias genéticas com as fendas. Matthews et al. ${ }^{3}$ encontra-

Setor de Medicina Fetal da Clínica Obstétrica do Hospital das Clínicas da Faculdade de Medicina da Universidade de São Paulo

Correspondência:

Victor Bunduki

Rua Oswaldo Moreira Pompeo, 61

04001-115 - São Paulo - SP ram 83\% de malformações associadas e 33\% de aneuploidias. Nicolaides et al. ${ }^{4}$ identificaram $48 \%$ de aneuploidias, ao passo que McGahan et al. ${ }^{5}$ observaram somente $10 \%$ de aberrações cromossômicas. Fischer et al. ${ }^{6}$ apresentaram a maior taxa de fendas faciais sem alterações outras nem aneuploidias (57\%).

A análise das fendas faciais no período prénatal diverge muito dos estudos realizados em serviços de cirurgias pediátrica e plástica, pois um número importante de fetos são portadores de anomalias graves com alta mortalidade, não sendo vistos pelos cirurgiões ${ }^{7}$.

Com a incorporação da ultra-sonografia na rotina propedêutica obstétrica, o diagnóstico prénatal das anomalias faciais fetais se tornou realidade, gerando situações nas quais o aconselhamento e conduta devem ser bem planejados.

O objetivo deste estudo foi avaliar os casos de fenda labial e/ou palatina diagnosticados no Setor de Medicina Fetal da Clínica Obstétrica da Faculdade de Medicina da Universidade de São 
Paulo quanto ao tipo de defeito, sua associação com fendas palatinas, a freqüência de outras malformações estruturais associadas e/ou de aberrações cromossômicas. O seguimento e a evolução pósnatal destes casos também são descritos.

\section{Pacientes e Métodos}

Durante o período de setembro de 1995 a dezembro de 1999, foram identificados 40 casos de fenda labial e/ou palatina no Setor de Medicina Fetal da Clínica Obstétrica da Faculdade de Medicina da Universidade de São Paulo.

Todos os casos foram submetidos a exames ultra-sonográficos nível III seriados (cada 3 a 4 semanas) e ecocardiografia fetal, além de estudo citogenético por meio da amniocentese ou cordocentese. A avaliação da integridade facial fetal foi realizada por corte coronal tangencial anterior da face evidenciando lábio superior, lábio inferior e nariz (Figura 1). Diante do achado de fenda labial lateral, a integridade do palato era avaliada mediante cortes transversais da face e crânio fetais no nível da arcada alveolar superior. O estudo da integridade do palato era, ainda, complementado por meio de cortes sagitais da face avaliando-se a presença ou não de fluxo transpalatino com o auxílio do Doppler colorido durante o gasping fetal $^{8}$.

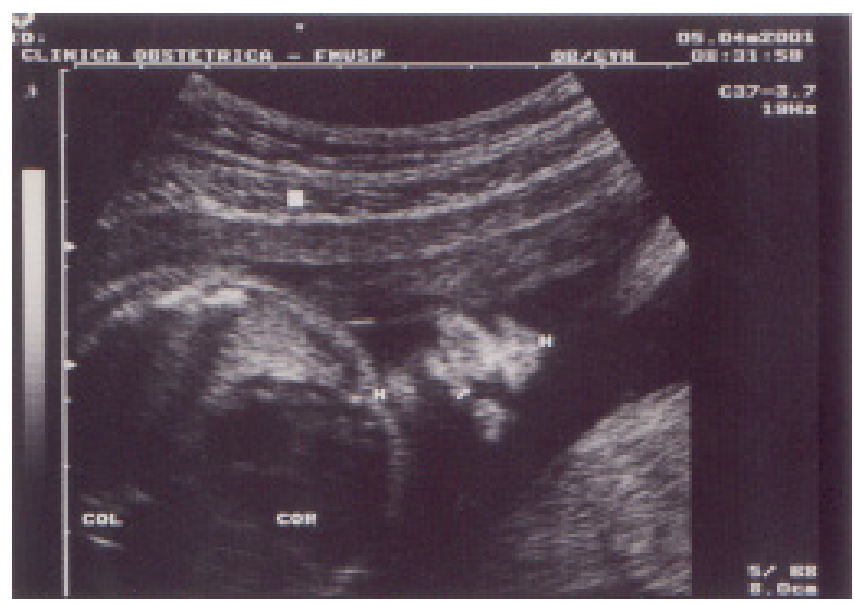

Figura 1 - Corte coronal da face fetal tangenciando os lábios e nariz (N), demonstrando fenda labial unilateral à esquerda (seta). M: mento; cor: coração; col: coluna.

Foram avaliados os seguintes parâmetros: idade gestacional no diagnóstico, idade materna e antecedentes de fenda labial e/ou palatina, presença de outras malformações estruturais associadas, presença de aberrações cromossômicas, lado da lesão (unilateral - direita ou esquer- da - ou bilateral), tipo de lesão (labial, palatina, ou labiopalatina), mortalidade e o seguimento pós-natal.

O seguimento pós-natal foi realizado pelo acompanhamento dos casos nascidos no próprio hospital ou por meio de contato por cartas ou telefonemas para os restantes quanto à sobrevida, e época de correção da lesão. A qualidade da correção da lesão e o seguimento fonoaudiológico das crianças não fez parte do escopo deste trabalho.

Este estudo foi realizado após ser aprovado pela Comissão de Ética em Pesquisa do Hospital das Clínicas e os dados estudados dos casos foram coletados com a permissão das gestantes. Além disso, as avaliações citogenéticas foram realizados após consentimento escrito de cada paciente.

\section{Resultados}

Quarenta casos de fenda labial e/ou palatina foram identificados durante o período do estudo. A mediana da idade gestacional no diagnóstico foi de 26 semanas (variando de 14 a 38) e a idade materna esteve entre 16 e 43 anos (média de 28,3 e mediana de 28,5). Apenas em um caso foi encontrado antecedente de fenda labial fetal. Não houve história de fenda labial e/ou palatina nas gestantes nem nos familiares dos pais nesta série.

Durante o exame ultra-sonográfico no prénatal, foram identificados 19 casos de fendas labiopalatinas $(47,5 \%), 3$ de fenda palatina (5\%) e 18 fetos com fendas labiais (45\%). Após o parto, observou-se também defeito do palato em 4 casos de fendas diagnosticados durante o pré-natal como puramente labiais (taxa de falso-negativo de $20 \%$ para o diagnóstico de fenda palatina associada à labial). Após o nascimento, com a confirmação do diagnóstico, observou-se que o sexo masculino predominou nos casos de fenda labial pura (9/15 $60 \%)$ e o sexo feminino foi mais afetado (15/25 $60 \%$ ) quando o palato esteve comprometido. Em relação ao local da lesão, 22 fetos (55\%) apresentaram fenda unilateral, localizada principalmente à esquerda (14/21 casos - 66,6\%), 10 (25\%) apresentaram fenda mediana e $8(20 \%)$ possuíam lesões bilaterais.

Em 10 casos (10/40 - 25\%) o diagnóstico ultra-sonográfico pré-natal mostrou fendas labiais e/ou palatinas isoladas, isto é, sem outras malformações fetais nem aneuploidias. Destes, observou-se lesão labial em 9 fetos (90\%), todos unilaterais, com predomínio de localização à esquerda (6/9 - 66,7\%), seguida pela lesão à direita (3/9 $33,3 \%)$. O sexo masculino predominou nestes casos (6/9). Em 1 caso (1/10 - 10\%) a fenda foi do 
tipo labiopalatina ao ultra-som durante o período pré-natal.

Quanto à avaliação após o nascimento, dos 9 casos que apresentavam fendas labiais puras ao ultra-som durante o período pré-natal, foi identificada lesão palatina associada em 3 recémnascidos. Estas lesões palatinas corresponderam a fendas do palato duro $(n=2)$ e da porção membranosa ( $\mathrm{n}=1$ ), que caracterizam malformações da união do palato secundário embrionário. A taxa de falso-negativo, neste grupo, para o diagnóstico de lesão palatina associada à fenda labial foi de 3/9 (33,3\%).

Este grupo apresentou sobrevida de 100\% e todos foram submetidos a cirurgia plástica reparadora em diferentes épocas (Tabela 1).

Tabela 1 - Características dos casos de fendas faciais isoladas quanto ao tipo e local da lesão, ao sexo fetal e à evolução clínica.

\begin{tabular}{|c|c|c|c|c|c|}
\hline $\mathrm{n}$ & Tipo de fenda & Local da fenda & Sexo & Confirmação pós-natal & Evolução \\
\hline 1 & L & $E$ & $\mathrm{~m}$ & S & Vivo, so \\
\hline 2 & L & $E$ & $f$ & $N(L P)$ & Vivo, SO \\
\hline 3 & L & D & $f$ & $N(L P)$ & Vivo, SO \\
\hline 4 & L & D & $\mathrm{m}$ & S & Vivo, So \\
\hline 5 & L & E & $\mathrm{m}$ & $S$ & Vivo, So \\
\hline 6 & LP & $\mathrm{E}$ & $f$ & $S$ & Vivo, So \\
\hline 7 & L & E & $\mathrm{m}$ & $S$ & Vivo, so \\
\hline 8 & L & E & $f$ & $S$ & Vivo, so \\
\hline 9 & L & E & $\mathrm{m}$ & $N(L P)$ & Vivo, so \\
\hline 10 & L & E & $\mathrm{m}$ & S & Vivo, so \\
\hline
\end{tabular}

n: número do caso; L: labial; LP: labiopalatina; E: esquerda; D: direita; m: masculino; f: feminino; S: sim; N: não; SO: cirurgia plástica reparadora

Outras malformações estruturais associadas foram observadas em 30 casos $(75 \%)$, sendo que aneuploidia foi identificada em 10/30 fetos $(33,3 \%)$. A Tabela 2 mostra as principais malformações associadas nos fetos com cariótipo normal, ao passo que a Tabela 3 mostra as características dos casos com anomalias cromossômicas. Neste grupo de fetos com malformações associadas (tanto com ou sem aneuploidia), a fenda labiopalatina foi diagnosticada em 18 casos (60\%), sendo 7 medianas, 7 bilaterais, 2 unilaterais à esquerda e 2 unilaterais à direita. A lesão foi somente labial em 9 fetos (30\%): 3 bilaterais, 3 unilaterais à direita, 2 unilaterais à esquerda e 1 mediana. A fenda palatina esteve presente em 3 (10\%) casos: 2 unilaterais à esquerda e 1 unilateral à direita, sendo que um destes apresentava micrognatia associada e o diagnóstico de síndrome de Pierre-Robin foi realizado após o nascimento (caso 25 da Tabela 2). Em um caso no qual o exame pré-natal tinha diagnosticado fenda labial pura, observou-se lesão palatina após o nascimento.

A taxa de mortalidade geral neste grupo foi 24/30 (80\%), sendo observados 12 óbitos fetais, 7 neomortos precoces, 2 neomortos tardios e 1 abortamento espontâneo. Em 2 casos foi indicada a interrupção da gravidez após autorização judicial.

\section{Discussão}

Com o avanço tecnológico, a avaliação da face fetal passou a ser etapa fundamental do exame ultra-sonográfico morfológico. Assim, as fendas labiais e/ou palatinas, que são as malformações faciais mais freqüentes passaram a ser reconhecidas por meio deste exame, o que implica aconselhamento pré-natal adequado às pacientes.

A incidência das fendas labiais e/ou palatinas identificadas durante a vida intra-uterina em uma população de baixo risco é de aproximadamente $0,2 \%$, ao passo que na população de risco, esta doença pode ser encontrada em torno de $8 \%{ }^{9,10}$.

A literatura é controversa a respeito da importância de se realizar o diagnóstico de fendas labiais ou labiopalatinas isoladas durante o periodo pré-natal. As principais razões citadas contra a sua prática incluem por vezes a falta de um diagnóstico preciso, o alto custo e o impacto emocional sobre os pais ${ }^{11}$. Porém, outros defendem a realização do diagnóstico pré-natal, devido à possibilidade de se propor a interrupção da gravidez nos casos em que há outras malformações associadas ou com anomalias genético-cromossômicas e mesmo para o planejamento da cirurgia plástica logo após o nascimento ${ }^{3}$. Além disso, a polêmica aumenta quando se considera a cirurgia reparadora fetal, que para alguns evitaria cicatrizes permanentes no futuro ${ }^{12}$. 
Tabela 2 - Características dos casos de fendas faciais associadas a outras malformações (com cariótipo normal) quanto ao tipo e local da lesão, ao sexo fetal e à evolução clínica. Os tipos de lesão foram confirmados após o nascimento.

\begin{tabular}{|c|c|c|c|c|c|}
\hline $\mathrm{n}$ & Tipo de lesão & Local & Sexo & Malformações & Evolução \\
\hline 11 & $\mathrm{~L}$ & $D$ & $\mathrm{~F}$ & Cerebral & ONP \\
\hline 12 & $\mathrm{~L}$ & $\mathrm{D}$ & M & Cerebral & Vivo; SO \\
\hline 13 & $\mathrm{~L}$ & $E$ & M & Cerebral & OF \\
\hline 14 & LP & $E$ & M & Cerebral, membros & OF \\
\hline 15 & LP & M & M & Cerebral, membros & OF \\
\hline 16 & LP & B & M & Cerebral, renal & ONP \\
\hline 17 & LP & B & M & Cerebral, facial, membros & OF \\
\hline 18 & LP & B & M & Cerebral, face, membros & ONP \\
\hline 19 & $\mathrm{P}$ & $E$ & $\mathrm{~F}$ & Cerebral, cardiopatia & OF \\
\hline 20 & LP & M & $\mathrm{F}$ & Renal & Vivo; SO \\
\hline 21 & LP & D & $\mathrm{F}$ & Cerebral & OF \\
\hline 22 & $\mathrm{~L}$ & M & $\mathrm{F}$ & Cerebral & Vivo; SO \\
\hline 23 & LP & $E$ & $\mathrm{~F}$ & Cardiopatia & Vivo; SO \\
\hline 24 & LP & B & M & Cerebral, cardiopatia & OF \\
\hline 25 & $\mathrm{P}$ & $E$ & M & Facial * & Vivo; so \\
\hline 26 & $\mathrm{~L}$ & $E$ & M & Cerebral, renal, cardiopatia, membros & ONP \\
\hline 27 & LP & B & $\mathrm{F}$ & Cerebral & ONT \\
\hline 28 & $\mathrm{~L}$ & $\mathrm{~B}$ & M & Cerebral, membros & $\mathrm{Ab}$ \\
\hline 29 & $P$ & D & $\mathrm{F}$ & Cerebral, membros & OF \\
\hline 30 & LP & B & $\mathrm{F}$ & Cerebral & Vivo; so \\
\hline
\end{tabular}

Tabela 3 - Características dos casos de fendas faciais associadas a outras malformações e com cariótipo anormal, quanto ao tipo e local da lesão, ao sexo fetal e à evolução clínica.

\begin{tabular}{|c|c|c|c|c|c|c|c|}
\hline$n$ & Tipo de lesão & Local & Cariótipo & Sexo & Malformações & Confirmação pós-natal & Evolução \\
\hline 31 & LP & M & trissomia 13 & $f$ & Cardiopatia, membros & S & OF \\
\hline 32 & LP & M & trissomia 13 & $f$ & Cerebral, facial, membros & $\mathrm{S}$ & OF \\
\hline 33 & $\mathrm{~L}$ & $\mathrm{D}$ & trissomia 18 & $f$ & Cerebral, torácica, cardiopatia & $\mathrm{S}$ & $A b$ \\
\hline 34 & LP & $\mathrm{B}$ & trissomia 21 & $f$ & Cerebral, facial & $\mathrm{S}$ & ONP \\
\hline 35 & $\mathrm{~L}$ & M & trissomia 13 & $f$ & Cardiopatia, membros & $\mathrm{S}$ & ONT \\
\hline 36 & L & M & trissomia 13 & $\mathrm{~m}$ & Cardiopatia & $\mathrm{S}$ & OF \\
\hline 37 & LP & M & 47, XXY & $\mathrm{m}$ & Cerebral & $\mathrm{S}$ & Ab judiciário \\
\hline 38 & LP & $\mathrm{D}$ & trissomia 18 & $\mathrm{~m}$ & Cerebral, renal, membros & $\mathrm{S}$ & OF \\
\hline 39 & LP & M & trissomia 13 & $f$ & Cerebral, facial & $\mathrm{S}$ & ONP \\
\hline 40 & LP & M & trissomia 13 & $f$ & Cerebral, renal & $\mathrm{S}$ & OF \\
\hline
\end{tabular}

n: número do caso; L: labial; LP: labiopalatina; D: direita; M: mediana; B: bilateral; m: masculino; f: femino; S: sim; OF: óbito fetal; ONP: óbito neonatal precoce; ONT: óbito neonatal tardio Ab: abortamento judiciário

A alta prevalência de fendas associadas a outras malformações ou mesmo cromossomopatias na presente série se deve ao fato de trabalharmos em centro terciário ou de referência, o que leva à seleção de casos mais complicados, o que é observado também por outros autores $^{3,4}$.

A mediana da idade gestacional no diagnós- tico na nossa amostra foi de 26 semanas, sendo similar ao observado por Fischer et al. ${ }^{6}$ (média de 27 semanas). Alguns autores ${ }^{13,14}$ propõem o diagnóstico mais precoce, ao redor da $14^{\mathrm{a}}$ semana de gestação, com o auxílio da ultra-sonografia transvaginal. Ao nosso ver, o diagnóstico tardio, como na nossa experiência, não prejudica a conduta diante das fendas isoladas, porém é prejudi- 
cial quando estamos diante de múltiplas malformações ou aneuploidias fetais. Assim, o diagnóstico mais precoce, apesar de gerar angústia para os casos de fenda isolada, é de fundamental importância quando malformações associadas estão presentes, situação em que o diagnóstico tardio prejudica o aconselhamento pré-natal ${ }^{15}$.

Khoury et al. ${ }^{16}$ indicam a idade materna maior de 35 anos como um fator de risco importante, o que poderia ser explicado pela maior possibilidade de aneuploidias, as quais estariam associadas às fendas faciais. Este fato não foi comprovado nem por Lopoo et al. ${ }^{17}$ nem pelos nossos resultados, pois a média geral da idade materna foi respectivamente de 30 e de 28 anos. Mesmo se considerarmos o grupo de pacientes com aneuploidias, a idade materna média foi de 29 anos.

Ao se considerar o grupo geral, nossa experiência está de acordo com a literatura quanto à distribuição do tipo de fenda e da sua localização, sendo as fendas labiais, principalmente unilaterais à esquerda, e as labiopalatinas as mais prevalentes, inclusive com pequena falha no diagnóstico de lesão palatina associada (em 4 casos deste estudo não se observou defeito do palato, além do labial). A fenda palatina pura, isto é, sem lesão do lábio associada, foi identificada em 3 casos, pois estes fetos apresentavam anomalias estruturais importantes e aneuploidia, o que motivou a avaliação exaustiva e cautelosa do lábio e palato fetais, culminando na identificação desta lesão.

A fenda labial, na nossa experiência, foi mais observada em fetos masculinos, ao passo que a lesão palatina foi mais comum no sexo feminino, o que também foi relatado por outros autores ${ }^{2}$. A explicação para este fato seria que a fusão dos palatos ocorre mais tardiamente no sexo feminino.

A associação de outras malformações com o diagnóstico de fenda labial e/ou palatina é comum, apesar de não se saber ao certo a percentagem real deste achado. Na nossa experiência, foi observada a mesma freqüência de malformações associadas que Lopoo et al. ${ }^{17}$ observaram (75\%).

No nosso estudo, o local e o tipo de fenda se distribuíram diferentemente nos diferentes grupos. Assim, dentre os fetos com fendas faciais isoladas, a fenda labial unilateral à esquerda foi a mais freqüente e não se observou lesão mediana. Em contrapartida, o grupo de fetos com malformações associadas e/ou aneuploidias apresentou predomínio das fendas labiopalatinas medianas e bilaterais. Esta observação vai ao encontro da ex- periência relatada de Benacerraf e Mulliken ${ }^{18}$, que descrevem ausência de aneuploidias nos casos de fendas unilaterais, ao passo que diante de lesões bilaterais e/ou medianas a taxa de aneuploidias foi relevante.

Devido ao fato de inúmeras doenças cromossômicas e gênicas cursarem com fendas faciais, estas, quando identificadas, servem como sinal de alerta para o médico ultra-sonografista, sendo indispensável a realização de minucioso exame morfológico fetal, além da ecocardiografia fetal. Segundo Nyberg et al. ${ }^{19}$, o cariótipo fetal deve ser considerado em casos em que há malformações associadas e/ou quando a fenda facial diagnosticada apresenta alto risco de cromossomopatia (lesões medianas ou bilaterais). Ao nosso ver, deve-se ainda, e por princípio básico em medicina fetal, oferecer o estudo do cariótipo fetal sempre que se identifica fenda facial, explicando os riscos e os benefícios do diagnóstico citogenético no período pré-natal. Deve-se ressaltar que casos em que se diagnosticam outras malformações associadas e/ou fendas medianas ou bilaterais, o benefício supera em muito os riscos destes procedimentos diagnósticos.

Apesar de as lesões labiais puras e unilaterais não terem cursado com aneuploidias fetais em nossa experiência e na de outros autores ${ }^{19,20}$ acreditamos ser ainda prematuro abandonar a indicação de cariótipo fetal diante destas lesões. Estas séries são, ao nosso ver, ainda não conclusivas quanto ao aumento de risco para aneuploidia fetal ligado à presença de fenda facial isolada.

Por meio deste estudo, pode-se observar que as fendas faciais estão freqüentemente associadas a outras malformações estruturais e mesmo aneuploidias. Assim, pela ultra-sonografia morfológica detalhada, deve-se pesquisar outras malformações e indicar o estudo do cariótipo fetal. As fendas isoladas estão associadas com excelente prognóstico.

Ainda que indicado por princípio nos fetos com fendas labiais unilaterais isoladas detectadas pela ultra-sonografia, é indiscutível que o estudo do cariótipo deva ser sempre realizado nos casos de fendas com lesões associadas e/ou fendas faciais medianas ou bilaterais.

Todos os fetos com diagnóstico de fenda facial devem, portanto, ser encaminhados para centros de referência para que uma cuidadosa avaliação pré-natal seja oferecida, a fim de se realizar o seguimento mais adequado possível destes casos. 


\section{SUMMARY}

Purpose: to evaluate fetuses with facial cleft as to type of lesion, associated malformations and aneuploidies.

Method: the following parameters were evaluated: maternal age and previous history, gestational age at diagnosis, lesion side, type of lesion, presence of associated malformations and aneuploidies, mortality rate and postnatal follow-up. Results: forty fetuses had facial cleft, 18 (45\%) cases had cleft lip, 19 (47.5\%) had cleft lip and palate, and $3(7.5 \%)$ cases presented with cleft palate. Isolated facial cleft was observed in 10 fetuses (25\%), all of them unilaterally located. Aneuploidies were identified in 10/30 (33.33\%) of the patients with associated malformations. Cleft lip and palate was more often seen in this group (18/30 - 60\%), followed by bilateral lesion (8/30 - 26.7\%) and median cleft (10/30 - 33.3\%).

Conclusion: facial clefts are considered excellent signs for the presence of associated malformations and fetal aneuploidies. Fetuses with facial cleft must be referred to specialized centers in order to have specialized ultrasound and genetic analysis which can provide the best prenatal counseling for these cases. Isolated facial cleft was associated with very good prognosis.

KEY WORDS: Cleft lip. Cleft palate. Prenatal diagnosis. Fetal malformations.

\section{Referências}

1. Melink M. Cleft lip and cleft palate: etiology and pathogenesis. In: Kernahan DA, Rosenstein SW, Dado DV, editors. Cleft lip and palate: a system of management. $4^{\text {th }}$ ed. Baltimore: Williams \& Wilkins; 1990. p.3-12.

2. Yetter JF. Cleft lip and cleft palate. Am Fam Physician 1992; 46:1211-21.

3. Matthews MS, Cohen M, Viglione M, Brown AS. Prenatal counseling for cleft lip and palate. Plast Reconstr Surg 1998; 101:1-5.

4. Nicolaides KH, Salvesen DR, Snijders RJ, Gosden CM. Fetal facial defects: associated malformations and chromosomal abnormalities. Fetal Diagn Ther 1993; 8:1-9.

5. McGahan J, Coates T. The fetal face. In: McGahan J, Porto M, editors. Diagnostic Obstetrical Ultrasound. $6^{\text {th }}$ ed. Philadelphia: JP Lippincott; 1994. p.207-29.
6. Fischer N, Perrotin F, Tranquart F, Robert M, Lansac J, Body G. Prise en charge des fentes labiales et/ou palatines diagnostiquées in utero. J Gynecol Obstet Biol Reprod (Paris) 1999; 28:446-55.

7. Elmendorf EN, D’Antonio LL, Hardesty RA. Assessment of the patient with cleft lip and palate. A developmental approach. Clin Plast Surg 1993; 20:607-21.

8. Monni G, Ibba RM, Olla G, Cao A, Crisponi G. Color Doppler ultrasound and prenatal diagnosis of cleft palate. J Clin Ultrasound 1995; 23:189-191.

9. Jones MC. Facial clefting: etiology and developmental pathogenesis. Clin Plast Surg 1993; 20:599-606.

10.Hafner E, Sterniste W, Scholler J, Schuchter K, Philipp K. Prenatal diagnosis of facial malformations. Prenat Diagn 1997; 17:51-8.

11.Hulnick SJ. Ultrasound diagnosis of clefts. Plast Reconst Surg 1982; 70:118-9.

12.Dado DV, Kernahan DA, Gianopoulos JG. Intrauterine repair of cleft lip: what's involved? Plast Reconstr Surg 1990; 85:461-7.

13.Bronshtein M, Blumenfeld I, Blumenfeld Z. Early prenatal diagnosis of cleft lip and its potential impact on the number of babies with cleft lip. Br J Oral Maxillofac Surg 1996; 34:486-7.

14.Merz E, Weber G, Bahlmann F, Miric-Tesanic D. Application of transvaginal and abdominal threedimensional ultrasound for the detection or exclusion of malformations of the fetal face. Ultrasound Obstet Gynecol 1997; 9:237-43.

15.Blumenfeld Z, Blumenfeld I, Bronshtein M. The early prenatal diagnosis of cleft lip and the decisionmaking process. Cleft Palate Craniofac J 1999; 36:105-7.

16. Khoury MJ, Gomez-Farias M, Mulinare J. Does maternal cigarette smoking during pregnancy cause cleft lip and palate in offspring? Am J Dis Child 1989; 143:333-7.

17.Lopoo JB, Hedrick MH, Chasen S, et al. Natural history of fetuses with cleft lip. Plast Reconstr Surg 1999; 103:34-8.

18.Benacerraf BR, Mulliken JB. Fetal cleft lip and palate: sonographic diagnosis and postnatal outcome. Plast Reconstr Surg 1993; 92:1045-51.

19.Nyberg DA, Sickler GK, Hegge FN, Kramer DJ, Kropp RJ. Fetal cleft lip with and without cleft palate: US classification and correlation with outcome. Radiology 1995; 195:677-84.

20.Houze de l'Aulnoit D, Ellart D, Furby F, Ghazi D, Brabant G, Delcroix M. Diagnostic échographique anténatal des fentes labiales et labio-palatines. J Gynecol Obstet Biol Reprod (Paris) 1991; 20:325-31. 